\title{
Information processing and timing mechanisms in vision
}

\author{
Andrea Guazzini ${ }^{1}$, Pietro Lió ${ }^{2}$, Andrea Passarella ${ }^{1}$, and Marco Conti ${ }^{1}$ \\ ${ }^{1}$ Institute of Informatics and Telematics - IIT - CNR, via G. Moruzzi,1 - 56124 \\ Pisa, Italy \\ \{andrea. guazzini, andrea. passarella, marco. conti\}@iit. cnr. it \\ ${ }^{2}$ Computer Laboratory, University of Cambridge, 15, J.J. Thompson Avenue, \\ Cambridge, CB30FD, UK \\ pl219@ cam. ac. uk
}

\begin{abstract}
Researches of neural mechanism of time perception is one of the fastest growing areas of neuroscience. The visual system presents several examples of timing mechanisms. Its activity is characterized by a complex network of synchronized elements which cooperate together. Some authors recently proposed that neural circuits should be inherently capable of temporal processing as a result of the natural complexity of cortical networks coupled with the presence of time-dependent network properties. We present an adaptive feedback model which, through a temporal-to-spatial transformation is able to explain recent experiments on the relationships between vision and time/space perception.
\end{abstract}

\section{Introduction}

A fundamental question about the perception of time is whether the neural mechanisms underlying temporal judgments are universal and centralized in the brain or modality specific and distributed. Some authors have recently hypothesized that the temporal scaling of visual experience and the temporal tuning properties of certain visual neurons may be linked [?]. The apparent duration of a dynamic stimulus can be manipulated in a local region of visual space by adapting to oscillatory motion or flicker [?]. Perceived time is compressed when stimuli are flashed shortly before or after the onset of a saccadic eye movement [?]. Neurons in visual areas of primate parietal cortex have reduced latencies to visual stimulation at the time of a saccade [?]. Recently one class of models, called state-dependent networks, has been developed in order to demonstrate that a neural circuits can be inherently capable of temporal processing as a result of the natural complexity of cortical networks coupled with the presence of timedependent network properties [?]. We present an adaptive feedback model which we show to provide a possible explanation of recent experiments and insightful clues in order to elucidate the relationships between stimulus neural encoding and time/space perception [?]. Our model can be defined as an adaptive feedback model because it changes the strength of network connections during its activity in order to adapt to the changing contexts during the process; modifyng retroactively its sensitivity to the subsequent stimuli. 


\section{Brain timing and time Perception}

From a neuropsychological point of view, interval timing in the range of milliseconds to minutes is affected in a variety of neurological populations involving disruption of the frontal cortex, hippocampus, basal ganglia and cerebellum. The conclusion is that representation of time depends on the integration of multiple neural systems [?].Models of time perception all agree that temporal information is processed in many ways: it is remembered, compared to other temporal information, and used in the production of motor outputs. Researchers have had great difficulty in pinning timing-related activity in the brain to any specific type of function. This is largely because most of the time measurement tasks draw upon more than one process, making it difficult to tease the various components apart [?]. Johnston et al. has shown that apparent duration of a dynamic stimulus can be manipulated in a local region of visual space by adapting to oscillatory motion or flicker [?]. This implies spatially localized temporal mechanisms in duration perception. The authors have not found concomitant changes in time of onset or offset of the test pattern, demonstrating a direct local effect on the time duration perception rather than an indirect effect on the time course of neural processing. Moreover many perceptive tasks do not require explicit encoding of time: perception of visual motion, for example, relies on the output of spatio-temporally tuned neurons rather than on independent estimates of space and time. While there is good evidence about different clocks for different interval lengths, some recent evidences point clearly to the existence of visually based timing mechanism [?]. Finally one of the most up to date experimental result concerns the existence of a large contrast effects in the discrimination of short temporal intervals. Della Rocca et al. examined the effect of temporal distractors on interval discrimination. Subjects compared the duration of the second interval in a three-flash sequence with the interval between a two-flash sequence. For short durations, the presence of the irrelevant distractor interval affected the apparent duration of the test duration in a contrasting manner: short distractors caused it to appear longer, and vice versa. For very short probe durations $(<100 \mathrm{~ms})$ the effect was large, changing the perceived duration by up to a factor of two. For longer probe durations the effect of distractors reduced steadily, to no effect for durations greater than $500 \mathrm{~ms}$. The results for visual flashes, auditory tones, and brief vibrations were similar, implying the existence of two mechanisms for timing brief events, one for short intervals (less than 500 $m s$ ), and another for longer intervals, and that the transition between the two is gradual [?]. One hypothesis drawn from the previous evidences is that cerebral circuits are inherently able to rescale durations in a proportional manner and compensate for the error differences generated by the cerebellum. With the motivation of modeling some of these experimental findings, we organize the paper as follows. In the next section we provide a description of the reasoning and mathematical formulation of the model. In section results we discuss the extensive simulations we have performed. Then the following section reports on conclusions. The software used for the model is available upon request from the first author. 


\section{The Model}

We have modeled a key mechanism for the encoding of the temporal characteristics of the neural visual stimuli. Our inspiration originates from works on neural and visual timing. Several hypothesis of time measuring systems, peculiar to neural circuits, demonstrates integration (synchronization) enables both the evaluation of different time scales and the binding between different sensorial/cognitive modalities. There is a general belief on the hypothesis of the existence of a representation of temporal information relative to visual stimuli. The fundamental elements of the representation of visual information should be able to encode temporal information similarly to the other characteristics of the percept (color, frequency, brightness). In a recent paper, Buonomano proposed that short-term plasticity and dynamic changes in the balance of excitatoryinhibitory interactions may underlie the decoding of temporal information, that is, the generation of temporally selective neurons [?]. He first showed that it is possible to tune cells to respond selectively to different intervals by changing the synaptic weights of different synapses in parallel. Short-term plasticity is a usage-dependent change in synaptic strength on the time scale of millisecond to seconds and is observed in almost every synapse types of the central nervous system. Each type of synapse has its own specificity with respect to this property. When stimulated a few times within a second, some synapses show facilitation, others depression or else complex sequences of facilitatory and depressing changes [?].

\section{Behavior of a chain of neurons: the neural delay chain}

A neuron that responds to a vertical bar or a $1 \mathrm{kHz}$ tone must receive functional inputs from the appropriate sensory neurons in the retina or cochlea, while temporal information refers to stimuli defined by the temporal structure of active sensory neurons. If a bar of light is present for 50 or $100 \mathrm{msec}$, in both cases the same groups of retinal ganglion cells are active. Similarly, if two brief $1 \mathrm{kHz}$ tones are separated by 50 or $100 \mathrm{msec}$, the same population of hair cells will be active. Thus, for neurons to respond selectively to a 50 or $100 \mathrm{msec}$ stimulus, an additional process such as a temporal to spatial transformation must occur. Several experimental evidences suggest that the neural signal encode "time related properties" by changing the state of the network's connections. Thus the key mechanism could rely with the propagation of the signal in the earlier states of the neural encoding process $(\lesssim 1 \mathrm{sec}$ ). This networks could be regarded as "neural delay chain", and be represented by a neural network where the signals propagates from the primary perceptive circuits. It is possible to identify a system characterized by computationally well-definable elements which represent the neural delay chain. In such system the microscopic elements may be characterized following the formalism of the neural networks typified by synapses with long -term plasticity, which tend to synchronize their activity. Consequently the activity may be treated at numerical level as continuous value instead of considering the discharge frequency of the single nodes. Therefore each node may 
be characterized by an activation value between 0 and 1 which represents the synchronization level of the node's neurons. Each node is connected through oriented synapses to the following node. Due to the short term plasticity property of synapses it is possible to represent their level of strengthening and their status after the passage of the neural spike. Figure ?? shows the effects of a propagation of a neural signal along a neural delay chain. In the upper part of the figure the ordinate represents the activity of the node, while in lower part the ordinate indicates each synaptic weight along the chain. The abscissa represents the neural chain which carry out the role of a temporal buffer where the propagation of the neural signal encodes time information about the stimulus. The elements $\left(x_{i}\right)$, defined simply "nodes", represent thus populations of neurons, and the activation value associated to each node $(\in[0,1])$ represents the synchronization status of the node's neurons network. This approach allows to study a neural delay chain using a continuous representation of the neural signal. The status of the connections of the node is explicitly taken into account the status of the connections of the nodes $\left(a_{i}\right)$. The neural spike generated by the visual stimulus propagates along the chain, encoding the temporal information about the stimulus. The activation state of this network could be read by output neurons in order to assess the duration of a stimulus or of an interval, as it happens for distances between objects and their size. In this way we obtain in our model a temporal to spatial transformation.

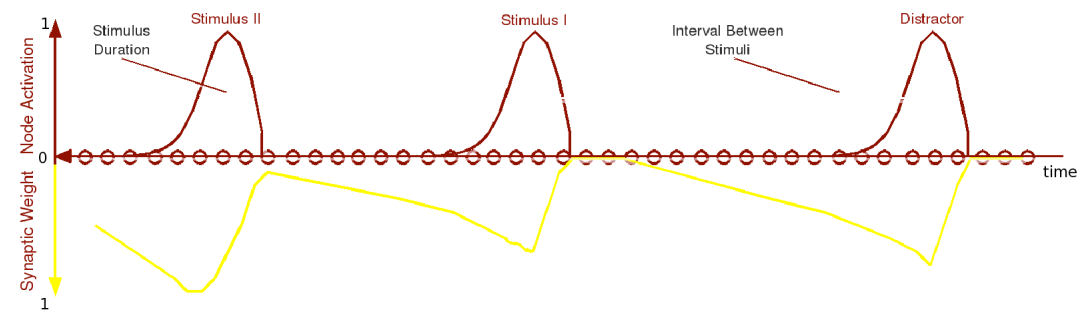

Fig. 1. The neural spike of the visual stimulus propagates along the neural delay chain (from left to the right) and encodes implicitly the time information about the stimulus. The activation state of this neural network is read out by specific neurons which assess the duration of a stimulus or of an interval, the same as it happens for distances among objects and their size.

We model a neural network where each node $(x)$ is characterized by an activity value (i.e. the state of synchronization) between 0 and 1 . Each node has excitatory synapses $(a)$ toward the following one. Synapses evolve their weight dynamically according to the connected nodes' activity. The coupled dynamical equations which describe the system evolution are characterized by three control parameters: A synaptic weight decay factor $(\alpha)$, a learning factor $(\gamma)$ which rules the synapses weight increment due to its activity, and a parameter which links the wave propagation velocity with the synaptic weight $(\theta)$. Each node within 
the delay chain must possess an activation function temporally correlated to the one of the preceding node. In this sense its activation must be proportional to the activation of the previous node properly weighted by the connection $\left(a_{i j}\right)$. Furthermore this equation determines the speed of the neural impulse as a function of $\theta$, as shown by the Figure ?? where the normalized wave velocity is indicated in the ordinate as a function of a fixed synaptic weights characterizing the neural delay chain. Specifically the value of activation of the node at the position $i+1$ at the time $t+1$ can be written as follows:

$$
x_{i+1}^{t+1}=x_{i}^{t}+\left(a_{i}^{t}\right)^{\theta}\left(x_{i}^{t}-K_{x}\right) .
$$

The synapse must enforce itself very rapidly depending on the signal coming from the preceding node and directed to the following one. The learning function of these oriented synapses must therefore be very sensitive to the activation changes of the following node and must be able as well to strengthen itself fast enough so as to facilitate the propagation of the activation wave. In order to approximate the non-linear features of the synaptic dinamics we use an hyperbolic tangent $([?])$, whose weight grows as a function of the learning factor $(\gamma)$ and the signal intensity. Finally the parameter $\varepsilon$ is here introduced to seed the dynamics and so driving its initial regime. The value of synapse $i$ at the time $t+1$ can be computed as follows:

$$
a_{i}^{t+1}=a_{i}^{t}+\tanh \left(\frac{\left(a_{i}^{t}-K_{a}\right)}{\left(1-K_{a}\right)}+\varepsilon\right) \cdot \gamma \cdot\left(x_{i}^{t}-K_{x}\right) .
$$

In Figure ?? is reported the value of a synaptic weight after the passage of neural signals with respect to the same value before the signal, for different stimulus duration. The return of the synapse to the rest condition (i.e. when no signals are exciting the synapse) should be sufficiently fast on its sensitiveness and in order to increase the precision of the system in the resolution of different signals. This dynamics can be modeled as follows:

$$
a_{i}^{t+1}=a_{i}^{t}+\alpha \cdot\left(a_{i}^{t}-K_{a}\right) .
$$

Since we are dealing with a symbolic representation of a biological system it is appropriate to define minimum activity thresholds for the elements of the system. So we will have a basic threshold of activity for the nodes $\left(K_{x}\right)$ and a rest value for the synapses $\left(K_{a}\right)$.

Our model relies on the growing and decay dynamics of the synaptic weights, and the interplay between these aspects and the propagation/diffusion of the neural signals. Consequently for simplicity we have explicitly separate the decay dynamics from the growing one.

$$
\left\{\begin{array}{l}
\text { if }\left(x_{i}^{t}-K_{x}\right)>0 \rightarrow \text { equation ?? holds, } \\
\text { if }\left(x_{i}^{t}-K_{x}\right)=0 \rightarrow \text { equation ?? holds, }
\end{array}\right.
$$

The first equation determines the synapses behaviour when no signal is arriving, in this case the decay rate is proportional both to the decay factor $\alpha$ 


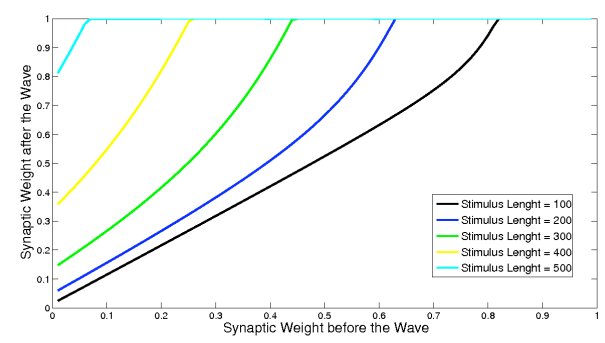

(a)

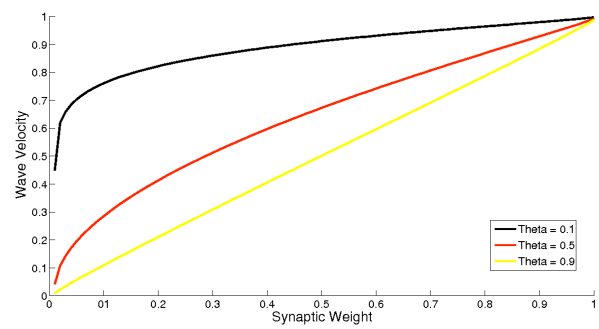

(b)

Fig. 2. (a)-Synaptic weight increase for different stimulus lengths; (b)-Normalized wave velocity for different values of the parameter which links the wave propagation velocity with the synaptic weight $(\theta)$.

and to the value of the synaptic weight itself. The second equation model the dynamics of synapses where a signal is arriving.

\section{Numerical simulation}

From the experimental data about the effects of a distractor on the perception of the time intervals, the existence of three distinct regimes is pointed out [?]. One where the distractor is too far to interfere with the couple of stimuli; a second where the distractor is very close to the first stimulus of the couple, thus causing an apparent dilatation of the perceived interval between the subsequent stimuli. Finally, a third regime where the distance of the distractor from the first stimulus is intermediate between the other two cases, and where a contraction of the perceived interval is observed. Our interpretation is that this phenomenon is generated by the nonlinearities which characterize the functions of the wave propagation, and of strengthening and relaxation of the network's connections. This interaction appears appropriate in certain regimes because it manages very well to solve different stimuli through the amplification of the differences between distant stimuli. Yet in certain regimes the same mechanism shows some constraints, among which there is the target of our model.

Following the experimental schema of Figure ??, we carried on numerical simulations. The experimental setting is simple enough to generate large amount 

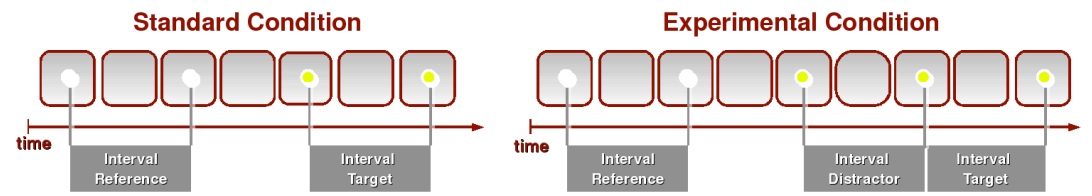

Fig. 3. In the experiment [?] simple visual stimuli were administered separated by interval of several lengths. In the standard condition (left) only a target and reference couples of stimuli were presented, while in the experimental condition (right) a distractor was administered before the first target stimulus. Interval between distractor and first stimulus has been manipulated in order to elucidate its effects. Finally the esteem of interval between target stimuli, obtained comparing it with the interval between reference stimuli, for both conditions has been compared.

of data which can be used to estimate model parameters with high accuracy. The model was composed by 20000 nodes and by the same number of synapses; we considered $\alpha=0.001, \gamma=0.01, \theta=0.1, \epsilon=0.01, k_{a}=0.1, k_{x}=0.1$, moreover the length of stimulus has been fixed to 400 time steps. When the stimulus is administered the chain is initialized setting the activation value of the first node of the chain to 1 . The first node remains at 1 for all the stimulus duration and it is set to the rest value $\left(k_{x}\right)$ when no stimulus is arriving. Control parameters have been varied between 1000 and 9000 time steps. The read-out process of the intervals from the neural chain is composed by two phases.In the first step those neuron which show the greater value of activation with respect to their neighbours are detected and labelled "local maxima". Afterwards the "distance" (e.g. the number of nodes) between those maxima of activations is assumed as the estimate of the target interval. The difference between the target interval estimate in the standard condition and in the experimental condition has been assumed as order parameter of the model.

\section{Results}

Numerical results are reported in Figure ?? and ??, on the vertical axis we put the difference between the evaluation of the interval between the target stimuli in presence of distractor, and the one in the control test. Moreover the abscissa and the ordinate of Figure ?? show the temporal intervals between the target stimuli (real gap) and between the distractor and the first target stimulus (distractor gap).The distortion caused by the distractor on the target interval estimate is qualitatively similar to [?]. When the distractor is near the first target stimulus a dilatation of the target interval is detectable with respect to the standard condition. The dilatation of perceived time is here caused by the different velocity of propagation of the signals which encode target stimuli. The first target signal propagates more quickly than the second one because it finds still excited synapses by the passage of the distractor stimulus. On the contrary for greater values of the target interval, when the distractor is enough distant from the first 


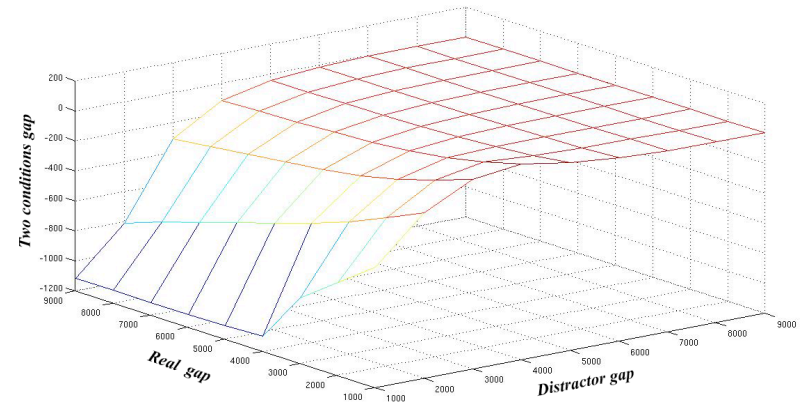

(a)

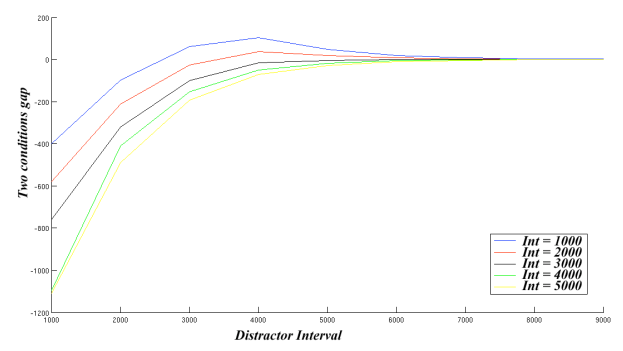

(b)

Fig. 4. Difference of target interval estimation (TIE) between reference and experimental conditions, indicated in the ordinate as "Two conditions gap", for different values of real and distractor gap. (a)- Surface in figure shows the effects of real and distractor gap on the TIE. (b)- Plot shows sections of the surface for different values of the variable real gap.

stimulus, a compression of the target interval emerges Figure ??. Consequently the compression is detectable when the second target signal encouters a medium (i.e. the state of the synapses) more excited than the first one. Noteworthy, see Figure ??, there are some appropriate values of the parameters of the model depending on which the model reproduces the experimental findings of Rocca et al [?]. In the model we observe that for extreme values of the distractor gap there is no detectable difference in the evaluation of the temporal interval. It is also interesting to note that for very low values of the distractor gap, i.e., when the distractor is presented immediately before the first target stimulus in temporal terms, the distortion in the precision of the evaluation of the interval between the two condition considerably diverges independently from the interval to evaluate (real gap). Finally it is possible to notice an interesting phenomenon which seems to provide confirmation of the qualitative phenomenology observed. In fact, for appropriate values of control parameters (real and distractor gaps) we achieve both the phenomenon of temporal compression and temporal dilatation. 


\section{Extension to neuron columns}

In our model the spikes travel along a chain of neurons. If one neuron in the chain becomes damaged or dies, the entire chain becomes ineffective. Given that neurons are dying during human aging, the natural selection has evolved mechanisms to avoid significant loss in information processing ability. It is now known the actual processors of the vision and other processes are cortical columns of neurons. Abeles and followers have proposed that cortical activities are based on two-dimensional neural networks whose connections are organised in a feedforward manner [?]. Localized waves of synchronous spiking activity travel along a sequence neurons organised in layers or pools [?]. If the number of excited neurons in a pool is above a threshold determined by the connectivity between layers, the wave activity initiated at the first pool propagates from one pool to the next, forming a synfire wave. The basic mechanism of trasmission relies on a trade-off of excitatory and inhibitory connections between neurons in consecutive pools; each neuron is receiving inputs by many neurons in the previous pool. The firing activity along a chain may propagate in either an asynchronous (i.e. a signal that reaches a threshold firing rate in one pool will cause an elevation in the firing rate in the next pool) or in a synchronous mode (i.e. a synchronous volley in one pool will elicit a synchronous volley in the other after one synaptic delay). Hertz showed that the only viable mode of transmission is the synchronous mode [?]. With these properties in mind, we considered an extension of the model to a single column of neuron and then to multiple columns. In the single column we generated and interconnected randomly sets of neurons, considering both inhibitory ad excitatory synapsis. The multiple column model considers the angle differences between neurons belonging to different pools. Considering single column neurons, we found results qualitatively similar to the single chain case. Work in progress focuses on multiple column case.

\section{Conclusion}

We present the model that reproduces the qualitative phenomena which characterized the experiment on using a distractor when measuring the visual timing ability. The real effect under scrutiny is here explained by a non linear behaviour of the neuron delay chain activation on the waves propagation velocity. The model could account also for the latency reduction found by Ibbotson et. al [?], explaining it as a consequence of changes occurred in the network activation state. Our model relies on local effects, because of the association of a neuron delay chain with each receptive field of the visual areas. Work in progress focuses on reproduce the real magnitude and time course of the considered phenomena.

Acknowledgments. We thank our collegues of Pisa Vision Lab for suggestions. This work is financed by the EU 6 Framework Programme Project: Measuring and Modelling Relativistic-Like Effects in Brain and NCSs. 


\section{References}

1. Abarbanel, H., Gibb, L., Huerta, R., and Rabinovich, M., Biophysical model of synaptic plasticity dynamics. Biol Cybern,(2003), 89, pp.214-226.

2. M. Abeles, Local Cortical Circuits, Berlin: Springer-Verlag, (1982).

3. Buonomano, D.V., Decoding Temporal Information: A Model Based on Short-Term Synaptic Plasticity, The Journal of Neuroscience, (2000), 20(3), pp.1129-1141.

4. Burr, D., Eye Movements: Keeping Vision Stable, The Current Biology, (2004), 14, pp.195-197.

5. Burr, D., Morrone, C., Perception: Transient Disruptions to Neural Space Time, The Current Biology, (2006), 16(19), pp.848.

6. Burr, D., Morrone, C., Time Perception: Space time in the Brain, Current Biology, (2006), 16(5), pp.171-173.

7. Dittman, J.S., Kreitzer, A.C., Regehr, W.G., Interplay between facilitation, depression, and residual calcium at three presynaptic terminals, Journal of Neuroscience, (2007), 20, pp.1374-1385.

8. Hertz, J. and A. Prugel-Bennett, Learning short synfire chains by selforganization, Network, (1996), 7, pp. 357-363.

9. Ibbotson, M., Crowder, N., Price, N., Neural basis of time changes during saccades, Current Biology, (2005), 16(19), pp.834-836.

10. Johnston, A., Arnold, D.H., Nishida, S., Spatially Localized Distortions of Event time, Current Biology, (2006), 16, pp.472-479.

11. Karmarkar, U., Buonomano, D., Timing in the Absence of Clocks: Encoding time in Neural Network States, Neuron, (2007), 53(3), pp.427-438.

12. Lewis, P.A. and Walsh, V., Time perception: components of the brain's clock, Current Biology, (2005), 15(10), pp.389-391.

13. Guazzini, A., Lio, P., Conti, M., Passarella, A., (2008). Complex network modeling of saccades control, Frontiers in Neuroinformatics. Neuroinformatics 2008. doi: 10.3389/conf.neuro.11.2008.01.039.

14. Meck, W.H., Neuropsychology of timing and time perception, Brain Cognition, (2005), 58(1), pp.1-8.

15. Rocca, E.D., Burr, D.C., Large contrast effects in the discrimination of short temporal intervals, Perception 36 ECVP, (2007).

16. Ross, J., Burr, D., Morrone, C., Suppression of the magnocellular pathway during saccades, Behavioural Brain Research, (1996), 80, pp.1-8.

17. Ross, J., Morrone, C., Goldberg, E., Burr, D., Changes in visual perception at the time of saccades, TRENDS in Neurosciences, (2001), 16, pp.472-479.

18. Wennekers, T, G.Palm: Controlling the speed of synfire chains. In: Malsburg C, Seelen W, Vorbruggen J, Sendhoff B (eds) Proceedings of ICANN 1996. Springer, 1996 